\title{
A Double Case of Nail-Patella Syndrome in the Same Family: The Importance of Nail Changes as Diagnostic Clues for Renal Involvement
}

\section{Michela Starace Ambra Di Altobrando Aurora Alessandrini Bianca Maria Piraccini}

Department of Experimental, Diagnostic and Specialty Medicine, Division of Dermatology, University of Bologna, Bologna, Italy

\section{Established Facts}

- Nail-Patella Syndrome is a rare disorder of autosomal dominant pleiotropic inheritance composed of the classic tetrad of fingernail dystrophy, patellar changes, dysplasia of the elbows, and presence of iliac horns. Less frequently, renal and ocular problems can be found.

- The diagnosis of this disorder is merely clinical, confirmed by iliac/patellar bone radiography.

- The clinical spectrum of Nail-Patella Syndrome is broad, with renal involvement representing the worst possible complication and determining the prognosis. Renal disease occurs in $30-50 \%$ of the affected individuals. Of these, less than $15 \%$ develop end-stage renal disease.

\section{Novel Insights}

- In our cases of Nail-Patella Syndrome, there was a significant discrepancy between the severity of nail changes and that of occult systemic involvement.

- It is fundamental to know and promptly recognize nail and skin changes of Nail-Patella Syndrome through a careful physical examination, since they can be pointers for the correct diagnosis and best management of possible severe renal manifestations.

\section{Keywords}

Nail · Nail pathology $\cdot$ Noninvasive diagnosis

\begin{abstract}
Nail diseases are rarely given significant importance in general consultation and their specific examination is often neglected, while localization to the nails could be an initial sign of a syndrome or a systemic disorder. Although rarely, nail alterations could be a sign of a systemic disorder, in which a prompt observation is crucial for an early diagnosis. We describe the case of an elderly woman with a long history of nail
\end{abstract}

dystrophy, previously labelled as nail fragility. On enquiry, similar nail changes were discovered to have affected the patient's son, who was visited the following week. Diagnosis of the Nail-Patella Syndrome (NPS) was suspected on the basis of nail alterations in both patients and confirmed by complementary radiographic examination. Screening for severe renal complications, which could have negatively affected the prognosis and have led to end-stage renal disease, followed. The prompt diagnosis of NPS and the associated diseases are mandatory for a correct management of these patients.

(c) 2019 S. Karger AG, Basel

\section{KARGER}

(c) 2019 S. Karger AG, Basel

E-Mail karger@karger.com

www.karger.com/sad
Ambra Di Altobrando, MD

Department of Experimental, Diagnostic and Specialty Medicine Division of Dermatology, University of Bologna, Via Massarenti, 1 IT-40138 Bologna (Italy)

E-Mail ambra.dialtobrando@studio.unibo.it 


\section{Introduction}

The examination of the nail should be an essential part of the physical examination. Correct clinical history and careful examination help the clinician to distinguish the different conditions and to decide the correct management of nail diseases. Indeed, nonspecific nail changes in combination with other skin and systemic manifestations can lead to a probable diagnosis of many genodermatoses [1].

The clinical spectrum of the Nail-Patella Syndrome (NPS) is broad, with nail involvement representing the most constant feature and renal involvement representing the worst possible complication and determining the prognosis. Renal disease occurs in $30-50 \%$ of the affected individuals. Typically, renal dysfunction appears slowly over many years and its severity can be different even within the same family [2].

We report the case of an elderly woman with a long history of nail dystrophy, previously labelled as nail fragility. On enquiry, similar nail changes were discovered to have affected the patient's son. Diagnosis of NPS was suspected on the basis of nail alterations in both patients and confirmed by complementary radiographic examination of the patellae and iliac bone. Screening for severe renal complications revealed initial renal failure in the son. We observed a discrepancy between the severity of nail changes and the presence of systemic involvement. In spite of nail alterations of mild cosmetic significance, causing minor discomfort for the patient, he in fact presented more severe occult renal manifestations, which could have affected his prognosis in case of delayed diagnosis.

\section{Case Report}

A 68-year-old woman was visited at our Outpatient Consultation for Nail Disease of the Dermatology Unit of the Department of Experimental, Diagnostic and Specialty Medicine (DIMES) of the University of Bologna complaining of nail deformity involving fingernails and some toenails. The nail alterations had been present since birth and had been previously labelled as nail fragility.

On examination, 9 out of 10 fingernails were affected and only the left fifth nail was spared. Nail changes were more evident on the ulnar side and the damage severity reduced progressively from the thumbnails to the little fingers. All affected nails presented Vshaped lunulae. The thumbnails and index nails showed the most evident alterations, since they appeared hypoplastic and dystrophic; moreover, the thumbnails were spoon-shaped and ridged longitudinally, while the index nails were spoon-shaped and split into two halves by a longitudinal cleft. The third and fourth nails presented fragility, a spoon-shape and a mild discoloration. The right fifth nail showed only a triangular lunula (Fig. 1a, b).
The patient also reported difficulty in gaining weight and was experiencing relapsing episodes of hip dislocation. Past medical history also included a worsened range of motion over the years. She also reported that her son presented similar dysplastic nails, but to a milder degree. Therefore, the 40 -year-old son was visited the following week to observe his nails. On examination, 8 out of 10 nails appeared affected. All 8 affected nails presented V-shaped lunulae. As with the mother, the most evident alterations involved the thumbnails and index nails. The thumbnails appeared dystrophic, thickened, longitudinally ridged and did not reach the free edge of the finger. Nails from the index to the fourth fingers were fragile, spoon-shaped and slightly discolored (Fig. 2a, b).

Adding to the clinical findings, the hypothesis of NPS was raised for both patients and complementary examinations were carried out to confirm the diagnosis.

Regarding the mother, X-rays of the knees revealed mild hypoplasia of both patellae, while X-rays of the hips presented evidence of previous dislocations. Regarding the son, X-rays of the knees revealed mild hypoplasia of both patellae, while no significant skeletal-muscle abnormalities were found on his hip radiographs.

Accordingly, some screening examinations were performed. Investigations on the mother did not reveal renal or ocular anomalies. Ocular investigation on the son was normal, while renal inquiry pointed out mild proteinuria and hematuria. A nephrological counseling was promptly requested.

The diagnosis of NPS was then confirmed. Genetic testing with sequence analysis did not find mutation of LMX1B genes. No further genetic testing was performed.

\section{Discussion}

Nail diseases are rarely given significant importance in general consultation and their observation is often overlooked by the clinician. However, nail alterations could be a sign of a systemic disorder or syndrome, in which a prompt observation is crucial for an early diagnosis of many genodermatoses [1].

NPS, also called onycho-osteo-arthrodysplasia, Osterreicher syndrome, Turner-Kieser syndrome or Fong's syndrome, is a rare disorder of autosomal dominant pleiotropic inheritance with variable expressivity and an estimated incidence of 1/50,000 individuals and without gender prevalence. Since the genetic mutation is localized on chromosome $9 q 34.1$, nail hypoplasia is associated with bone and kidney abnormalities [2,3].

NPS is composed of the classic tetrad of fingernail dystrophy, patellar changes, dysplasia of the elbows and presence of iliac horns. Less frequently, renal and ocular problems can be found [4-7]. The diagnosis of NPS is merely clinical, but the genetic testing for LMX1B mutation can be considered in uncertain cases [6-9].

Clinical manifestations change from person to person in terms of frequency and severity. The diagnosis could 
Fig. 1. Nail changes due to Nail-Patella Syndrome in the 68-year-old mother. a Nail changes are more evident on the ulnar side and damage severity progressively reduces from the thumbnails to the little fingers. V-shaped lunulae are present in all fingernails. The thumbnail and index nail are hypoplastic, the thumbnail is spoonshaped and ridged longitudinally, while the index nail is spoon-shaped and split into two halves by a longitudinal cleft. b Onychoscopy reveals the characteristic finding of the triangular lunula.

Fig. 2. Nail changes due to Nail-Patella Syndrome in the 40-year-old son. a As for the mother, the most evident alterations involve the thumbnail and index nails, but to a milder degree. b Onychoscopy of the index nail, partially split into two halves by a longitudinal cleft, reveals a triangular lunula.
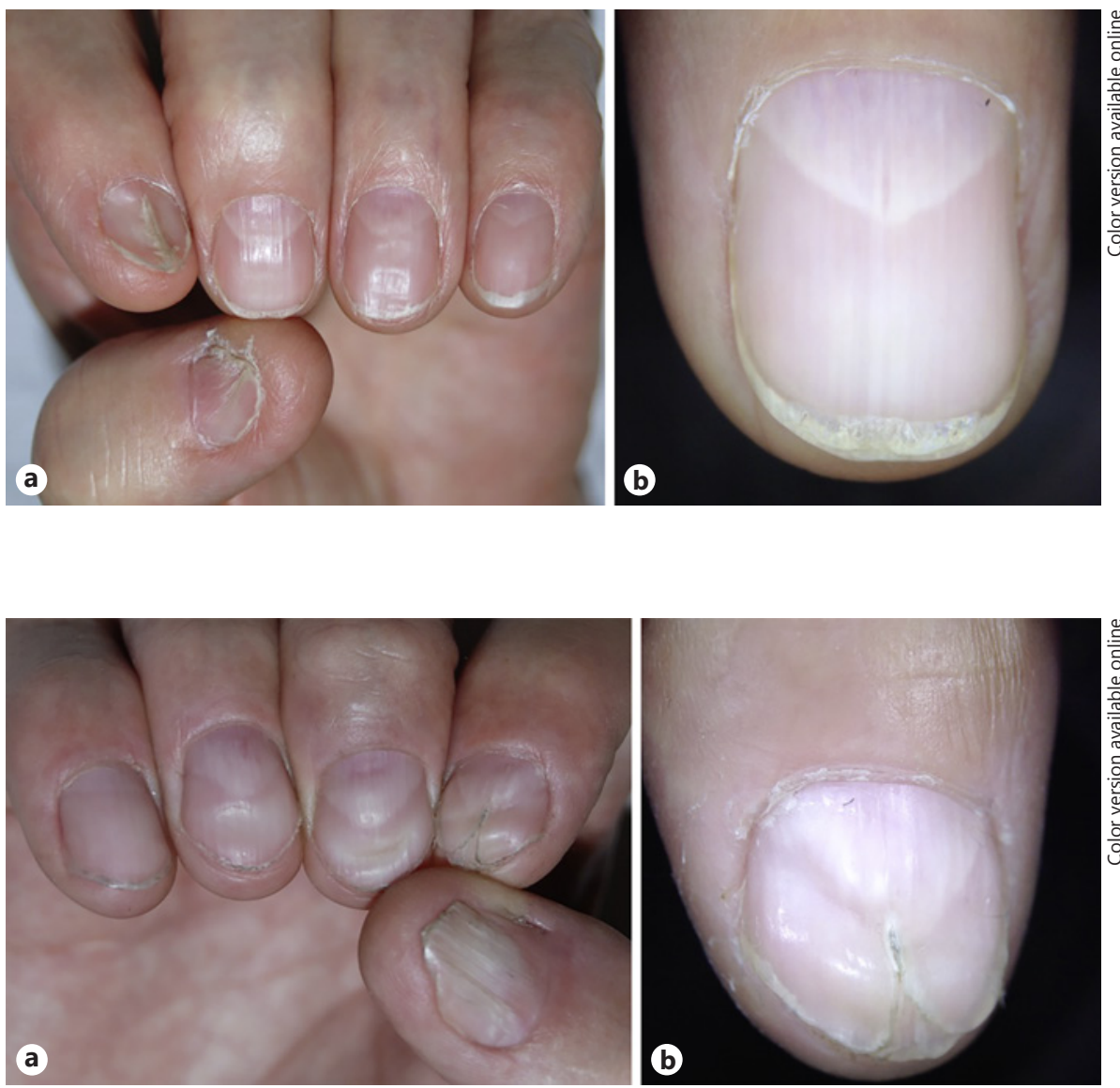

be done at birth, but it is often delayed. The two pathognomonic signs of NPS are the triangular/V-shaped appearance of the lunula and iliac horns, consisting in asymptomatic symmetrical bony formations arising from the posterior and lateral part of the iliac crests. However, many other manifestations have been described as well [5, $7,9]$.

The most constant feature of NPS is nail involvement (98\%), even if it can be strikingly variable. Fingernails are more commonly affected than toenails and abnormalities are often bilateral and symmetrical. Nail changes are classically more severe on the ulnar side of the thumbs and become less noticeable towards the fifth finger. Nails can be absent or appear hypoplastic/dystrophic and typically do not reach the free edge of the finger; they can be fragile, spoon-shaped, longitudinally or horizontally ridged, pitted, discolored, split into two halves by a longitudinal cleft or a ridge of skin, thin or thickened $[4,5,9]$. The triangular shape to the lunula is a unique finding, which can be better recognized by onychoscopy: the lunulae of the affected fingernail have a pointed tip directed distally [10].
The loss of skin creases on the dorsal aspects of the distal interphalangeal joints is another possible sign of NPS that is less known but very significant since its severity is correlated to the severity of nail changes [5].

Bone abnormalities in NPS may be asymmetrical and can be observed in more than $70 \%$ of patients. The patellae may be hypoplastic, fragmented or absent; elbow joint anomalies may comprise limitation of movements, cubitus valgus, antecubital pterygium (elbow contracture with webbing) $[5,9]$.

Other clinical features of NPS include spinal and chest wall disorders, osteoporosis, a tall and slender body habitus, neurologic alterations such as paresthesia and reduced sensation to pain and temperature, gastrointestinal symptoms such as constipation or irritable bowel syndrome, ophthalmologic involvement with primary openangle glaucoma, intra-ocular hypertension and iris pigmentary changes (Lester's sign) $[5,7,8]$.

The clinical spectrum of NPS is broad, with renal involvement representing the worst possible complication and determining the prognosis $[4,7,9]$. Renal disease oc- 
curs in $30-50 \%$ of the affected individuals. Of these, less than $15 \%$ develop end-stage renal disease. Typically, renal dysfunction appears slowly over many years and their severity can be different even within the family. The first signs of renal failure are hematuria and proteinuria, which can decrease spontaneously, remain asymptomatic or rapidly progress to nephrotic syndrome and to endstage renal disease $[3,5,7]$.

In our patients, in regard to the son, there was discrepancy between the severity of nail changes and that of systemic involvement. In spite of nail alterations of cosmetic significance, causing minor discomfort for the patient, he presented more severe occult manifestations elsewhere, which could have affected his prognosis in case of delayed diagnosis. As a result of the possible multisystemic manifestations, it is fundamental to know and rec- ognize nail and skin changes of NPS through a careful physical examination, since they can be pointers for the correct diagnosis and best management.

\section{Statement of Ethics}

All procedures followed were in accordance with the ethical standards of the responsible committee on human experimentation (institutional and national) and with the World Medical Association Declaration of Helsinki. Subjects have given their consent to publish details and photos of the case.

\section{Disclosure Statement}

The authors do not have any conflict of interest to declare.

\section{References}

1 Inamadar AC, Palit A. Nails: diagnostic clue to genodermatoses. Indian J Dermatol Venereol Leprol. 2012 May-Jun;78(3):271-8.

2 McIntosh I, Dreyer SD, Clough MV, Dunston JA, Eyaid W, Roig CM, et al. Mutation analysis of LMX1B gene in nail-patella syndrome patients. Am J Hum Genet. 1998 Dec;63(6): $1651-8$.

3 Bongers EM, Gubler MC, Knoers NV. Nailpatella syndrome. Overview on clinical and molecular findings. Pediatr Nephrol. 2002 Sep;17(9):703-12.
4 Neri I, Piccolo V, Balestri R, Piraccini BM, Patrizi A. Median nail damage in nail-patella syndrome associated with triangular lunulae. Br J Dermatol. 2015 Dec;173(6):1559-61.

5 Sweeney E, Hoover-Fong JE, McIntosh I. Nail-Patella Syndrome. In: Adam MP, Ardinger HH, Pagon RA, Wallace SE, Bean LJ, Stephens K, et al., editors. GeneReviews ${ }^{\circledR}$. Seattle: University of Washington; 2003. p. 1993-2019.

6 Kamath S, Bhagwandas K. Nail-patella syndrome with an emphasis on the risk of renal and ocular findings. Pediatr Dermatol. 2010 Jan-Feb;27(1):95-7.
7 Witzgall R. Nail-patella syndrome. Pflugers Arch. 2017 Aug;469(7-8):927-36.

8 Curbo ME, Park KJ, Brown LD, Incavo SJ. Total knee arthroplasty in a patient with nail-patella syndrome (NPS). Knee. 2019 Jan;26(1):273-78.

9 Agarwal S, Divecha C, Tullu MS, Deshmukh CT. A rare case of nephrotic syndrome: 'nailed' the diagnosis. J Postgrad Med. 2014 Apr-Jun;60(2):179-82.

10 Starace M, Alessandrini A, Piraccini BM. Nail disorders (onychoscopy). In: Lallas A, Errichetti E, Ioannides D, editors. Dermoscopy in general dermatology. Boca Raton: CRC Press; 2018. 